\title{
Tics und Tourette-Störung für die Praxis
}

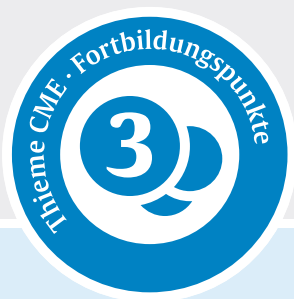

Anaïs Djodari-Irani ${ }^{1}$, Christos Ganos ${ }^{2}$

1 Klinik für Psychiatrie und Psychotherapie, Charité - Universitätsmedizin Berlin

2 Klinik für Neurologie, Charité - Universitätsmedizin Berlin

\section{ZUSAMMENFASSUNG}

Primäre Tic-Störungen und das Tourette-Syndrom (TS) sind häufige neuropsychiatrische Erkrankungen, die sich im frühen Lebensalter manifestieren und durch motorische und/ oder vokale Tics gekennzeichnet sind. Oftmals bestehen bei den Betroffenen psychiatrische Komorbiditäten wie Zwangsstörungen, Aufmerksamkeitsdefizit-/Hyperaktivitätsstörung, Angst- und affektive Störungen. Differenzialdiagnostisch stellen neben anderen Bewegungsstörungen wie zum Beispiel
Chorea oder Stereotypien, die funktionellen Tic-ähnlichen Störungen eine besondere Herausforderung dar. Tiermodelle, Post-mortem-Studien, behaviorale und bildgebende Studienbefunde weisen auf Veränderungen im kortiko-striato-thalamo-kortikalen Regelkreis in der primären Tic-Störung und dem TS hin, wobei in der Genese dieser Erkrankungen das dopaminerge System eine wichtige Rolle zu spielen scheint. Therapeutisch werden spezifische verhaltenstherapeutische Strategien wie das Habit Reversal Learning oder das Exposure and Response Prevention in erster Linie empfohlen. Pharmakotherapien beinhalten antipsychotische Medikation, Botulinumtoxin-Injektionen sowie cannabisbasierte Medikamente. In therapieresistent verlaufenden Fällen kann die tiefe Hirnstimulation erwogen werden.
Primäre Tic-Störungen und das Tourette-Syndrom (oder Tourette-Störung laut DSM-5, „Tourette disorder“, TS) sind neuropsychiatrische Erkrankungen, die im Kindesalter beginnen und sich bei ca. $1 \%$ der Patienten bis ins Erwachsenenalter zeigen [1]. Männer sind häufiger betroffen als Frauen (m:w=4:1, [2]). Die Zeit bis zur Diagnosestellung beträgt meist 5 bis 10 Jahre. Dieser Beitrag gibt einen Überblick über Symptomatik, Differenzialdiagnosen, Pathophysiologie und Therapie dieser häufigen neuropsychiatrischen Erkrankung. Detaillierte Einblicke in die Themen klinisches Spektrum und Komorbiditäten, Pathophysiologie sowie Therapieoptionen finden sich in den Artikeln des vorliegenden Hefts.

\section{Was sind Tics}

Tics, die zu den Hyperkinesien zählen, sind wiederholte, meist kurze Bewegungen, oft in rascher Folge, die häufig monomorph ablaufen und affektiv verstärkt werden [3]. Im Unterschied zu anderen Hyperkinesien, wie Myoklonus oder Chorea, geht bei der Mehrheit der Betroffenen, vor allem ab der Adoleszenz, dem Tic ein Vorgefühl („premonitory urge“) voraus [4]. Dieses wird häufig als unwiderstehlich empfunden - als „Drang, eine Bewegung auszuführen“, als körperlicher oder psychischer „Druck“ und etwas seltener als „Brennen“, „Kribbeln“ oder „Spannung “ beschrieben. So können Menschen mit Tics meist erkennen, dass ein bestimmter Tic eintreten wird. Bei diesen stellt somit die Tic-Ausführung eine willkürliche Konsequenz des unwillkürlichen Vorgefühls dar, die meist zu einer kurzfristigen Entlastung führt. Im Gegensatz zu an- deren Hyperkinesien, wie zum Beispiel Myoklonus, wird der Betroffene von Tics nicht überrascht.

Ein weiteres Charakteristikum von Tics ist die willentliche Unterdrückbarkeit, die bei fast allen Betroffenen, wenn auch für eine sehr kurze Dauer, möglich ist [5]. So kann Kontrolle über die Tics ausgeübt werden, die meist zu einer Verstärkung des Vorgefühls führt und somit nur temporär durchzuhalten ist. Interessanterweise können Betroffene während Phasen von Tic-Unterdrückung willkürliche Handlungen regulär ausführen. Somit stellt die willkürliche Tic-Kontrolle einen besonderen inhibitorischen Mechanismus dar, der spezifisch auf Tics und nicht auf das gesamte motorische Verhalten wirkt [6].

\section{Tic-Phänomenologie und klassische Charakteristika}

Die Tics werden in motorische oder vokale Tics sowie in einfache oder komplexe (Beteiligung mehrerer Muskelgruppen mit Ähnlichkeit zu willkürlichen Handlungen) Tics unterteilt [7] (Kasten). Deren Auftreten zeigt meist eine spezifische Somatotopie; so sind der Kopf/Schulter-Bereich sowohl am häufigsten und als auch am schwersten betroffen [5]. Des Weiteren zeigen Tics in primären Tic-Störungen (TS, chronisch motorische oder vokale Tic-Störung, vorübergehende (ICD-10) bzw. vorläufige (DSM-5) Tic-Störung) typischerweise Fluktuationen („waxing and waning“). Dieses wird gekennzeichnet durch Änderungen in Intensität und Frequenz, sowohl im Tagesverlauf als auch in längeren Intervallen. So berichten meist 
Kinder und Adoleszente, dass Tics in Stressphasen (z. B. Prüfungszeiten) zunehmen, um dann wieder beim Nachlassen psychosozialer Stressoren (z. B. im Urlaub) zurückzugehen. Tics sind darüber hinaus suggestibel und lassen sich teilweise durch externe Stimuli auslösen, zum Beispiel, wenn in der Sprechstunde über spezifische Tics gesprochen wird. Es ist jedoch auch nicht ungewöhnlich, wenn der Patient mit Tic-Störung von geringerer Schwere im Rahmen der ersten Sprechstundenkontakte nur sehr wenig Tics zeigt. Diese Patienten berichteten häufig über ein „Austicken“ nach erfolgtem Arztkontakt, d. h. wenn die Anspannung nachlässt.

\section{UNTERTEILUNG}

\section{Beispiele für einfache und komplexe Tics}

Einfache motorische Tics

- Augen blinzeln, zwinkern

- Nase verziehen, rümpfen

- Mund öffnen, verziehen

- Grimassieren

- Zähne klappern

- Kopf schütteln, verdrehen, zucken

- Schultern zucken

- Arm-/Handbewegungen

- Rumpfbewegungen

- Bauchbewegungen

- Bein-/Fußbewegungen

\section{Komplexe motorische Tics}

- Scheinbar absichtsvolle Bewegungen, Gesten an Hand, Armen, Rumpf, Fuß, Beinen, im Gesicht

- Klatschen, klopfen

- verbiegende, beugende Rumpfbewegungen

- ausfahrende Armbewegungen

- Echopraxie (nicht zweckgebundene Imitation von beobachteten Bewegungen anderer Personen)

- Kopropraxie (obszöne Gesten: Mittelfinger zeigen, in den Schritt fassen)

- Palipraxie (selten): Wiederholen eigener Bewegungen

\section{Einfache vokale Tics}

- Räuspern

- Schniefen

- Husten, Hüsteln

- geräuschvolles Ein- und Ausatmen

- Quietschen, Grunzen

- Pfeifen, Summen

- Ausstoßen von Schreien

- Ausrufen von Silben (hm, ah, ha, eh)

- Ausstoßen von (Tier)lauten

\section{Komplexe vokale Tics}

- Echolalie (nicht zweckgebundene Wiederholung von gehörten Wörtern, Silben, Sätzen)

- Koprolalie (Ausrufen obszöner Wörter)

- Palilalie (unwillkürliches Wiederholen von selbst Gesprochenem)

- Sprechblockaden

- Ausrufen von Satzfragmenten

- Ausrufen von sozial unangemessenen Wörtern („non-obscene socially inappropriate behavior“, NOSI, z.B. „dumm, dumm”, „hallo, hallo“)

\section{Tourette-Syndrom}

Die Diagnosestellung des TS setzt mindestens 2 motorische und einen vokalen Tic mit Auftreten vor dem 18. Lebensjahr voraus, wobei die Tics jeweils nicht gleichzeitig vorkommen müssen. So reicht beispielsweise ein vokaler Tic in der Vergangenheit für die Diagnosestellung aus. Der Kasten gibt einen Überblick über die Klassifikation der primären Tic-Störung nach ICD-10 sowie DSM-5. Zur Diagnosestellung gehört immer eine ausführliche Anamnese sowie eine klinische Untersuchung der Phänomenologie und möglicher psychiatrischer Komorbiditäten. Bei ca. 90 \% aller Patienten mit TS bestehen psychiatrische Komorbiditäten, die immer mitevaluiert und ggf. mitbehandelt werden sollten. Hierbei sind die häufigsten Komorbiditäten AD(H)S, Zwänge, Ängste, Impulskontrollstörungen und im Erwachsenenalter zudem Depression und Suchterkrankungen.

\section{ÜBERBLICK KLASSIFIKATION}

\section{Primäre Tic-Störung nach ICD-10}

- F95.0: vorübergehende Tic-Störung (Dauer<12 Monate), kommt nur bei Kindern vor

- F95.1: chronische motorische oder vokale Tic-Störung (Dauer> 12 Monate, keine Remission länger als 2 Monate, nur motorische oder nur vokale (seltener) Tics, erste Tics vor dem 18. Lebensjahr

- F95.2: Gilles-de-la-Tourette-Syndrom: kombinierte Tic-Störung, mindestens ein vokaler Tic und multiple motorische Tics

- F95.8: sonstige Tic-Störungen

- F95.9: Tic-Störungen, nicht näher bezeichnet

\section{Tic-Störung nach DSM-5}

Tourette-Störung 307.23

- sowohl motorische als auch mindestens ein vokaler Tic

- die Frequenz der Tics zeigt sich zu-, abnehmend (waxing and waning) im Laufe der Zeit, sind aber für über ein Jahr nach Symptombeginn persistent 
- Symptombeginn vor dem 18. Lebensjahr

- Symptome können nicht auf Substanzkonsum (z. B. Kokain) oder andere Erkrankung (z. B. Chorea Huntington, postvirale Encephalitis) zurückgeführt werden

Persistierende motorische oder vokale Tic-Störung 307.22

- Motorische(r) oder vokale(r) Tic(s)

- die Frequenz der Tics zeigt sich zu-, abnehmend (waxing and waning) im Laufe der Zeit, sind aber für über ein Jahr nach erstem Symptombeginn persistent

- Symptombeginn vor dem 18. Lebensjahr

- Symptome können nicht auf Substanzkonsum (z. B. Kokain) oder andere Erkrankung (z. B. Chorea Huntington, postvirale Encephalitis) zurückgeführt werden

- die Diagnosekriterien haben auch im Laufe der Erkrankung die der Tourette-Störung nie erfüllt

\section{Vorläufige Tic-Störung 307.21}

- Motorische(r) und/oder vokale(r) Tic(s)

- der Tic/die Tics zeigen sich weniger als ein Jahr nach Symptombeginn

- Symptombeginn vor dem 18. Lebensjahr

- Symptome können nicht auf Substanzkonsum (z. B. Kokain) oder andere Erkrankung (z. B. Chorea Huntington, postvirale Encephalitis) zurückgeführt werden

- die Diagnosekriterien erfüllen die der Tourette-Störung bzw. der persistierenden motorischen oder vokalen Ticstörung nicht

\section{Was Tics nicht sind}

Von Tics abzugrenzen sind in der Praxis vor allem die funktionellen Tic-ähnlichen Bewegungen [8-11]. Hierbei handelt es sich laut ICD-10 um dissoziative Störungen (Konversionsstörungen), die das klinische Bild von Tic- bzw. Tourette-Patienten zu imitieren scheinen. Obwohl nach ICD-10 ein überzeugender zeitlicher Zusammenhang zwischen Auftreten der Symptome und belastenden Lebensereignissen vorliegen muss, ist ein solcher Zusammenhang nach DSM-5 für die Diagnosestellung nicht erforderlich. Somit beruht diese auf der Erkennung positiver klinischer Zeichen.

Cave: In vielen Fällen liegt bei funktionellen Tic-ähnlichen Bewegungen der Symptombeginn nach dem 18. Lebensjahr. Vorgefühl und Unterdrückbarkeit werden häufig verneint, ebenso besteht kein waxing and waning der Symptome, sondern eine gleichförmige Symptompräsentation oder eine allmähliche Intensitätszunahme von monomorphen Tic-ähnlichen Bewegungen. Komplexe Tics sind häufiger als einfache Tics und eine somatotopische Zuordnung wie beim TS liegt nicht immer vor. Die Betroffenen berichten meist über negative Familienanamnese in Bezug auf Tic-Störungen. Es bestehen komorbid gehäuft Erkrankungen wie eine posttraumatische Belastungsstörung (PTBS), affektive und Persönlichkeitsstörungen. Eine ausführliche Darstellung der klinischen Unterscheidungsmerkmale und möglicher Überlappungen ist im Artikel von Hübl und Ganos in diesem Heft (S. 284) zu finden. Zu betonen ist, dass, wie bei Patienten, die zeitgleich an epileptischen und nicht epileptischen Anfällen leiden, bei Betroffenen mit primären Tic-Störungen funktionelle Tic-ähnliche Bewegungen vorkommen können. Die Erkennung dieser Phänomene stellt eine Herausforderung dar [11].

\section{Entstehung von Tics und TS}

Die Pathogenese des Tourette-Syndroms ist nicht vollständig geklärt. Eine zentrale Rolle in der Entstehung der Erkrankung ist den Basalganglien sowie der kortiko-striato-thalamo-kortikale Regelkreis zuzuschreiben [12]. Tiermodelle für Tic-Störungen, neuropathologische Post-mortem-Studien sowie strukturelle und funktionelle bildgebende Daten zeigen multiple Veränderungen im Bereich des Striatums sowie im Konnektivitätsprofil primärer und sekundärer sensomotorischer Areale [12]. Kognitionspsychologische bzw. wahrnehmungspsychologische Ansätze gehen zunächst von einer gestörten inhibitorischen Kontrolle aus, in welcher eine Imbalance GABA-erger striataler Neurone angenommen wird. Des Weiteren implizieren behaviorale und bildgebende Studien eine veränderte, wahrscheinlich pathologisch erhöhte dopaminerge Transmission, die mit verstärktem Gewohnheitslernen („habit formation“) einhergeht $[13,14]$. So neigen Betroffene mit TS in behavioralen Testungen dazu, vermehrt habituierte Verhaltensmuster zu entwickeln, die sich während einer antidopaminergen Tic-Therapie normalisieren. Hierzu passend ist die „theory of event coding“, welche besagt, dass sowohl Wahrnehmungen als auch Handlungen gemeinsam in „event files“ gespeichert werden und in enger Verbindung stehen. Es gibt erste experimentelle Hinweise, dass bei Gilles-de-la-Tourette-Syndrom-Betroffenen die Kopplung zwischen Wahrnehmung und Handlung besonders stark ausgeprägt ist und Tics aufgrund dieser starken Bindung durch verschiedene Stimuli ausgelöst werden könnten [15]. Mehr Informationen zu dieser Thematik findet sich in dem Artikel von Kleimaker et al. (S. 291) in diesem Heft.

\section{Therapie}

Die Mehrheit der Menschen, die Tics aufweisen, sind sich über die Symptomatik nicht bewusst, da die repetitiven Bewegungen oder Phoneme zu ihrem regulären Verhaltensrepertoire gehören. Auch gibt es Betroffene, die sich der Symptomatik bewusst sind, sich jedoch durch diese nicht gestört fühlen. Ein weiterer Teil der Betroffenen empfindet das Vorhandensein der Tics als störend und ist dadurch so eingeschränkt, dass ärztliche Hilfe aufgesucht wird. Neben 
der Stigmatisierung, die Tics in der Öffentlichkeit hervorrufen können, führen sie auch nicht selten zu chronischen Schmerzen (häufig im HWS- oder im Kieferbereich) und manchmal auch zu Verletzungen („maligne Tics“). In diesen Fällen sollten Tics behandelt werden [16].

Da das TS und die chronische motorische Tic-Störung im Erwachsenenalter teilweise stark invalidisierende Erkrankungen sind, ist eine adäquate Therapie für die Patienten von enormer Wichtigkeit. Dabei ist der Patient darüber aufzuklären, dass die Erkrankung durch keine der heutigen Therapieansätze geheilt werden kann - lediglich können die Symptome unterdrückt werden. Ebenso sollte bedacht werden, dass die Patienten oft ihre Aufmerksamkeit auf die Tic-Reduktion richten müssen, um nicht zu stören. Dieses kann sehr häufig zu einer sich verschlechternden Leistung in Schule und Universität führen. Eine umfassende Beratung zu einer Einrichtung eines Grad der Behinderung oder eines Nachteilsausgleichs in Schule, Ausbildung und Studium sollte unbedingt erfolgen.

Nach einer ausführlichen Psychoedukation sollte eine spezifische Verhaltenstherapie im ersten Schritt empfohlen werden, da diese bereits eine Symptomreduktion von ca. $30 \%$ verspricht [17]. Eine „unspezifische“ Verhaltenstherapie, tiefenpsychologische oder analytische Therapie versprechen hingegen keine Symptomreduktion. In der Praxis ist es jedoch leider fast unmöglich, entsprechend geschulte Psychotherapeuten zu finden, die entsprechend der europäischen [18] und amerikanischen Leitlinien [19] First-line-Therapiekonzepte des Habit Reversal Training (HRT) oder Exposure and Response Prevention (ERP) beherrschen [17]. Beim Habit Reversal Training soll das Ausführen eines zuvor erlernten alternativen Verhaltens das Eintreten von Tics vermindern. Dafür wird zunächst ein Selbstwahrnehmungstraining durchgeführt, welches den Betroffenen die Tic-Expression und das damit assoziierte Vorgefühl bewusster werden lässt. In einem weiteren Schritt werden im Competing Response Training (Training inkompatibler Reaktionen) dann konkurrierende, körperlich nicht mit der Tic-Ausführung kompatible Verhaltensweisen identifiziert und erlernt, die die Betroffenen daran hindern, den Tic auszuführen. Die verhaltenstherapeutische Intervention CBIT (Comprehensive Behavioral Intervention for Tics) umfasst neben HRT auch psychoedukative Elemente, Entspannungstechniken sowie eine Analyse von (Umgebungs)-Faktoren, die die Tics verstärken und das entsprechende Erlernen eines entsprechenden Umgangs mit diesen Faktoren.

Durch die Therapie des ERP soll der oftmals von den Patienten beschriebene Automatismus unterbrochen werden, dass auf das Vorgefühl immer ein Tic folgen muss. Hierfür soll, auf der Lerntheorie basierend, zunächst eine Tic-Unterdrückung eingeübt werden und der Patient zunehmend mit Vorgefühl und Tic-auslösenden Umgebungsfaktoren konfrontiert werden, um den Druck, einen Tic ausführen zu müssen, zunehmend besser zu tolerieren und somit die Tic-Ausführung zu reduzieren. Führt die verhaltensthera-
- Tab. 1 Beispiele der medikamentösen Therapie von Tics

\begin{tabular}{|l|l|l|}
\hline Substanz & Startdosis $\mathbf{( m g )}$ & $\begin{array}{l}\text { Empfohlene Höchstmenge } \\
\mathbf{( m g )}\end{array}$ \\
\hline Aripiprazol 1 tgl. & 1 & $10-15$ \\
\hline Risperidon 1-2 tgl. & 0,25 & $2-3$ \\
\hline Olanzapin 1-2 tgl & 2,5 & $5-10$ \\
\hline Tiaprid 3 tgl. & $50-100$ & $300-600$ \\
\hline Sulpirid 2 tgl. & $50-100$ & 300 \\
\hline Dronabinol 3-4 tgl. & $\begin{array}{l}\text { 1 Tropfen 2,5\%iges } \\
\text { Dronabinol }\end{array}$ & $4-$ mal 6 Tropfen \\
\hline & & \\
\hline
\end{tabular}

peutische Maßnahme nicht zu einer ausreichenden Tic-Reduktion (oder in der Praxis die Regel: kann kein entsprechender Therapeut gefunden werden), sollte eine medikamentöse Therapie eingeleitet werden.

Hierzulande sind bei den erwachsenen Patienten die atypischen Antipsychotika die Mittel der ersten Wahl. Diese führen bis zu einer ca. 50 \%igen Tic-Reduktion. Am nebenwirkungsärmsten ist dabei das Aripiprazol, ein partieller Dopamin-D2-Rezeptoragonist. Bei Kindern gilt als Erstlinientherapie die Gabe des Benzamids Tiaprid. Auch mit Sulpirid wurden gute Erfahrungen gemacht. In der Praxis sind diese aber in der Tic-Reduktion den Antipsychotika deutlich unterlegen und schlechter verträglich. In den angloamerikanischen Ländern gehören die alpha-2-adreneregen Agonisten Clonidin und Guanfacin zu den Erstlinientherapeutika. Diese noradreneregen Wikstoffe sind aber nur in Studien mit Kindern mit starker ADHS-Komorbidität den Antipsychotika überlegen und mit deutlich mehr Nebenwirkungen behaftet als die bei uns gängigen Antipsychotika. \ Tab. 1 gibt einen Überblick über die in Deutschland am häufigsten verwendeten Therapeutika und ihre Dosierung, umfassendere Informationen finden sich in dem Artikel von Beste und Roessner in diesem Heft (S. 300).

Cave: Hierbei ist zu beachten, dass die Höchstdosierungen in der Tic-Behandlungen geringer ausfallen als in der Behandlung von psychotischen Erkrankungen. Auch ist auf ein langsameres Einschleichen antipsychotischer Medikation zu achten, um die unerwünschten Wirkungen möglichst gering zu halten. Häufig wird durch die Patienten geäußert, dass sie keine Tic-Reduktion unter der eingenommenen Medikation verspürt hätten, obwohl es durchaus einen messbaren Rückgang der Tic-Expression gibt.

Bei spezifischen Tics wie z. B. dystonen Tics im Schultergürtelbereich oder kranialen Tics wie Blinzeln, gibt es die Möglichkeit, Botulinumtoxin intramuskulär zu injizieren, um diese Tics zu reduzieren. Zunehmend wird der Wunsch nach „natürlichen“ Mitteln deutlich und die Patienten erfragen gezielt Cannabis-basierte Medikamente. Studien konnten zeigen, dass diese (z. B. Dronabinol - delta-9-Te- 
trahydrocannabinol oder Nabiximols Sativex) einen Tic-reduzierenden Effekt hatten und häufig zudem die psychiatrischen Komorbiditäten wie ADHS, Ängste und Depression lindern können. Studien beschäftigen sich zudem mit dem Cannabinoidmodulator ABX-1431, welches den Endocannabinoidspiegel erhöhen soll.

Sollte ein Patient diverse Antipsychotika nicht vertragen haben oder diese Therapie nicht ausreichend Tic-reduzierend gewesen sein, kann ein Antrag bei der Krankenkasse zur Kostenübernahme einer cannabisbasierten Therapie erwogen werden. Diese wird jedoch noch meist von den Krankenkassen abgelehnt. Genauere Informationen zu dem Thema der cannabisbasierten Medikamente in der Tic-Therapie finden sich in dem Artikel von Müller-Vahl (S. 314).

Darüber hinaus steht die Therapieoption der tiefen Hirnstimulation (Deep Brain Stimulation, DBS) im Rahmen von Studien zur Verfügung. Diese Option sollte bei noch inkonsistenten Stimulationstargets und -parametern nur den schwersten Fällen (z. B. bei Selbstmutilation) vorbehalten sein. Hier ist auf eine strikte Einhaltung der Ein- und Ausschlusskriterien, auf die kritische Prüfung der zu stimulierenden Zielareale und die Wahl eines erfahrenen Zentrums zu achten (siehe Artikel von Marcelino et al. S. 305).

\section{Fallbeispiel 1}

Frau D., 21 Jahre alt, Studentin der Kulturwissenschaft, stellt sich eigeninitiativ in der Tourette-Sprechstunde vor und gibt an, seit 3 Monaten unter diversen und täglich an Stärke zunehmenden Tics (Grimassieren, gegen den Kopf schlagen, Fiepen) zu leiden. In der Kindheit und Jugend habe sie nie unter Tics gelitten. Die Tics hätten sich plötzlich nach einem Streit mit dem Ex-Partner eingestellt - das initiale Phänomen sei ein Kopftremor gewesen. Nach 3 Tagen hätten sich Tic-ähnliche Bewegungen wie Grimassieren, gegen den Kopf Schlagen und Fiepen, gezeigt. Sie habe bereits eine Psychotherapie in der Jugend absolviert, da sie sich vom 12. bis 16. Lebensjahr selbst verletzt habe (Schneiden im Bereich der Unterarme zum Spannungsabbau). Im längeren Gespräch treten multiple Stressoren (Trennung vom psychisch und körperlich missbrauchenden Freund vor 4 Monaten, in Kindheit Gewalterfahrung durch alkoholabhängigen, an Schizophrenie erkrankten Vater) zutage. Frau D. kann bereits im ersten Gespräch die Verdachtsdiagnose einer funktionellen Störung annehmen und lässt sich auf einen psychosomatischen Klinikaufenthalt ein. Nach Diagnosestellung wandelt sich das Beschwerdebild und es zeigt sich vornehmlich ein Handtremor. In der Klinik für Psychosomatik (Schwerpunkt funktionelle Bewegungsstörungen) wird neben der Diagnose einer dissoziativen Störung die Diagnose einer PTBS gestellt und eine spezifische Psychotherapie im ambulanten Setting gebahnt. Bei der Wiedervorstellung nach 4 Monaten ist symptomatisch nur noch ein leichtes Grimassieren zu sehen. Frau
D. berichtete über einen deutlichen Rückgang der Symptomatik. Bei einer weiteren Vorstellung nach 8 Monaten sind die Tic-ähnlichen Symptome unter fortgeführter ambulanter Psychotherapie komplett verschwunden. Bei einer Einschlafstörung wird Quetiapin 25 mg bei Bedarf eingenommen, ansonsten ist keine Medikation notwendig.

\section{Fallbeispiel 2}

Herr D., ein 24-jähriger Berufsschüler, stellt sich erstmalig in der Tourette-Sprechstunde vor. Er habe einen Film gesehen, in dem ein Mann unter dem Tourette-Syndrom gelitten habe. Er frage sich nun, ob er auch an dieser Erkrankung leide. Schon mit 4 Jahren seien seinen Eltern „nervöse Zuckungen im Gesicht" aufgefallen, in der Jugend sei ein Räuspern hinzugekommen. Der Kinderarzt habe den Eltern gesagt, dass der Sohn „ein nervöser Typ“ sei. Wegen eines ADHS-Verdachts habe er in der Grundschulzeit eine Ergotherapie gemacht. Medikamentös sei er noch nie behandelt worden. Er leide zudem unter Zwangsgedanken, die teilweise quälenden Charakter hätten (u. a. Gedanken an Amputation der eigenen Füße). Seine Mutter habe mehrere Zwänge (z. B. Ordnungs- und Waschzwang), Tics habe in seiner Familie aber niemand. In der späten Jugend seien die Tics für einige Jahre weniger geworden. Nun merke er eine Zunahme der Gesichtszuckungen, die er sich nicht erklären könne. Zudem zeige er statt eines Räusperns ein Grunzen. Ab und zu verspüre er ein Vorgefühl und gehe dann teilweise auf die Toilette, „um es unbemerkt rauszulassen“. Er verspüre Scham bei der Benutzung der öffentlichen Verkehrsmittel und merke, dass er auf der besuchten Berufsschule die Mitschüler mit seinem Grunzen störe und verärgere. Seine ganze Konzentration müsse er für die Unterdrückung der Tics aufwenden, seine Noten würden immer schlechter. Ab und zu konsumiere er THC, da er bemerkt habe, dass dies die Tics reduziere. Insgesamt ziehe er sich mehr und mehr zurück und sei häufig gedrückter Stimmung.

Herr D. stimmt nach ausführlicher Psychoedukation und Aufklärung über die möglichen Nebenwirkungen einer Therapie mit Aripiprazol zu, da in Heimatnähe keine spezifische Verhaltenstherapie vorhanden ist. Nach erfolgtem unauffälligem EKG und unauffälligen Blutwerten beginnt die Therapie in langsam aufsteigender Dosierung. Bei einer Dosis von 10 mg gibt Herr D. an, kaum noch Tics zu verspüren, die Medikation vertrage er soweit gut. Nach erfolgter Antipsychotikaeinstellung verbleiben die Konzentrationsschwierigkeiten in der Berufsschule in reduziertem, aber immer noch störendem Umfang, weshalb eine Therapie mit Methyphenidat begonnen wird, die der Patient ebenfalls gut verträgt. Verbleibend und mit einem mittelschweren depressiven Syndrom verbunden sind die vor allem bei Stress zunehmenden quälenden Zwangsgedanken, weshalb eine Escitalopramgabe begonnen und eine Verhaltenstherapie initiiert wird. Die Therapeutin gab an, sich mit Habit Revearsal Training bzw. Exposure and Response Prevention nicht auszukennen, aber mit dem Pati- 
enten an einem verbesserten Stressmanagement arbeiten zu wollen. Nach einem Jahr ist der Patient unter Aripiprazol $10 \mathrm{mg}$ und Methyphendiat $40 \mathrm{mg}$ nahezu Tic-frei und berichtete über einen ungestörten Berufsschulbesuch. Die Zwangsgedanken sind unter Verhaltenstherapie und Escitalopram $20 \mathrm{mg}$ zumindest leicht rückläufig.

\section{FAZIT}

Zusammengefasst handelt es sich bei der primären Tic-Störung und dem TS um recht häufige neuropsychiatrische Erkrankungen, die sich im frühen Lebensalter manifestieren und durch motorische und/oder vokale Tics gekennzeichnet sind. Oftmals bestehen bei den Betroffenen psychiatrische Komorbiditäten wie Zwangsstörungen, $A D(H) S$, Angst- und affektive Störungen, die ggf. einer Mitbehandlung bedürfen. Die Differenzialdiagnose besteht in den funktionellen Tic-ähnlichen Störungen. Die therapeutischen Optionen für die primäre Tic-Störung sowie für das TS erstrecken sich von spezifischen verhaltenstherapeutische Strategien (z. B. Habit Reversal Learning oder Exposure and Response Prevention) über antipsychotische Medikation, Botulinumtoxin-Injektionen, cannabisbasierte Medikamente und in schwersten therapierefraktären Fällen die DBS.

\section{Interessenkonflikt}

\section{Erklärung zu finanziellen Interessen}

Forschungsförderung erhalten: nein; Honorar/geldwerten Vorteil für Referententätigkeit erhalten: nein; Bezahlter Berater/interner Schulungsreferent/Gehaltsempfänger: nein; Patent/Geschäftsanteile/Aktien (Autor/Partner, Ehepartner, Kinder) an Firma (Nicht-Sponsor der Veranstaltung): nein; Patent/Geschäftsanteile/Aktien (Autor/Partner, Ehepartner, Kinder) an Firma (Sponsor der Veranstaltung): nein.

Erklärung zu nicht finanziellen Interessen

Die Autoren geben an, dass kein Interessenkonflikt besteht.

\section{Korrespondenzadresse}

\author{
Dr. med. Anaïs Djodari-Irani \\ Klinik für Psychiatrie und Psychotherapie \\ Charité - Universitätsmedizin Berlin \\ Charitéplatz 1, Bonhoefferweg 3, 10117 Berlin \\ Tel. 030/450517095, Fax 030/450517953 \\ anais.djodari-irani@charite.de
}

\section{Literatur}

[1] Ganos C, Martino D. Tics and tourette syndrome. Neurol Clin 2015; 33: 115-136
[2] Yang J, Hirsch L, Osland S. The prevalence of diagnosed tourette syndrome in Canada: A national population-based study. Psychiatry Research 2017; 250: 228-233

[3] Meige H, Feindel EnCL, Wilson SAK. Tics and their treatment. London: S. Appleton, 1907

[4] Kwak C, Dat Vuong K, Jankovic J. Premonitory sensory phenomenon in Tourette's syndrome. Mov Disord 2003;18: 1530-1533

[5] Ganos C, Bongert ], Asmuss L et al. The somatotopy of tic inhibition: Where and how much? Mov Disord 2015;30: 1184-1189

[6] Ganos C, Rothwell J, Haggard P. Voluntary inhibitory motor control over involuntary tic movements. Mov Disord 2018. doi: $10.1002 / m d s .27346$

[7] Jankovic J. Tourette syndrome. Phenomenology and classification of tics. Neurol Clin 1997;15: 267-275

[8] 8. Baizabal-Carvallo JF, Jankovic J. The clinical features of psychogenic movement disorders resembling tics. J Neurol Neurosurg Psychiatry 2014; 85: 573-575

[9] Demartini B, Ricciardi L, et al. A positive diagnosis of functional (psychogenic) tics. Eur J Neurol 2015; 22: 527-e536

[10] Ganos C, Edwards M], Muller-Vahl K. “I swear it is Tourette's!”: On functional coprolalia and other tic-like vocalizations. Psychiatry Res 2016; 246: 821-826

[11] 11. Ganos C, Martino D, Espay AJ et al. Tics and functional tic-like movements: Can we tell them apart? Neurology 2019; 93: 750-758

[12] Ganos C, Roessner V, Munchau A. The functional anatomy of Gilles de la Tourette syndrome. Neurosci Biobehav Rev 2013; 37 : 1050-1062

[13] Palminteri S, Lebreton M, Worbe Y et al. Dopamine-dependent reinforcement of motor skill learning: evidence from Gilles de la Tourette syndrome. Brain 2011; 134: 2287-2301

[14] Delorme C, Salvador A, Valabregue R et al. Enhanced habit formation in Gilles de la de la Tourette syndrome. Brain 2016; 139: 605-615

[15] Beste C, Tubing J, et al. Altered perceptual binding in Gilles de la Tourette syndrome. Cortex 2016; 83: 160-166

[16] Ganos C, Martino D, Pringsheim T. Tics in the Pediatric Population: Pragmatic Management. Mov Disord Clin Pract 2016; 4: 160-172

[17] Fründt O, Woods D, Ganos C. Behavioral therapy for Tourette syndrome and chronic tic disorders. Neurology Clinical practice 2017; 7: 148-156

[18] Verdellen C, van de Griendt J, Hartmann A et al. European clinical guidelines for Tourette syndrome and other tic disorders. Part III: behavioural and psychosocial interventions. Eur Child Adolesc Psychiatry 2011; 20: 197-207

[19] Pringsheim T, Okun MS, Muller-Vahl K et al. Practice guideline recommendations summary: Treatment of tics in people with Tourette syndrome and chronic tic disorders. Neurology 2019; 92: 896-906

\section{Bibliografie}

DOI https://doi.org/10.1055/a-1133-6915

Nervenheilkunde 2020; 39: 320-325

(c) Georg Thieme Verlag KG Stuttgart · New York

ISSN 0722-1541 
Punkte sammeln auf CME.thiemede

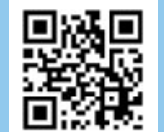

Diese Fortbildungseinheit ist bis zu 12 Monate online für die Teilnahme verfügbar. Den genauen Einsendeschluss finden Sie unter https://cme.thieme.de/CXERH2S.

Sollten Sie Fragen zur Online-Teilnahme haben, finden Sie unter https://cme.thieme.de/hilfe eine ausführliche Anleitung. Wir wünschen viel Erfolg beim Beantworten der Fragen!

Unter https://eref.thieme.de/CXERH2S oder über den QR-Code kommen Sie direkt zur Startseite des Wissenstests.

VNR 2760512020158722286

\section{Frage 1}

Welche Aussage trifft zu?

A Tics und Myoklonus können klinisch nicht unterschieden werden.

B Tics sind häufig langsame Bewegungen.

C Tics sind willkürlich unterdrückbar.

D Klonische Tics sind viel seltener als komplexe dystone Tics.

E Tics werden immer bewusst wahrgenommen.

\section{Frage 2}

Welche Aussage trifft nicht zu?

A Tics zeigen eine spezifische somatotopische Verteilung.

B Der Kopf/Schulter-Bereich ist am seltensten betroffen.

C Tics fluktuieren zeitlich sowohl in Bezug auf Intensität als auch auf Frequenz.

D Tics sind suggestibel.

E Häufig können Tics über längere Zeiten unterdrückt werden.

\section{Frage 3}

Welche Aussage trifft zu?

A Ein dopaminerges Defizit liegt der Pathophysiologie der Tics zugrunde.

B Tics sind Fragmente von epileptischen Bewegungen.

C Die Basalganglien spielen eine entscheidende Rolle bei der Entstehung von Tics.

D Antidopaminerge Substanzen führen typischerweise zu einer Verschlechterung von Tics.

E Routine EEG-Untersuchungen bei Menschen mit Tics zeigen spezifische Veränderungen.

\section{Frage 4}

Welche psychiatrische Komorbidität ist häufig bei Betroffenen mit primärer Ticstörung/Tourette-Syndrom?

A Paranoid-halluzinatorisches Syndrom

B Schizoaffektive Störung

C Zwangsstörung

D Posttraumatisches Belastungssyndrom

E Narzisstische Persönlichkeitsstörung

\section{Frage 5}

Welche Aussage trifft zu?

A Funktionelle Tic-ähnliche Bewegungen können leicht von Tics unterschieden werden.

B Tics und funktionelle Tic-ähnliche Bewegungen haben dieselbe Pathophysiologie.

C Alle Tics haben einen psychogenen Ursprung.

D Antidopaminerge Therapien sollten in erster Linie auch bei funktionellen Tic-ähnlichen Bewegungen angewendet werden.

E Die Unterscheidung zwischen Tics und funktionelle Tic-ähnliche Bewegungen kann zum Teil sehr schwierig sein.

\section{Frage 6}

Beim Tourette-Syndrom ist zur Diagnosestellung notwendig das Vorliegen von ...

A mindestens einem vokalen und einem motorischen Tic.

B mindestens 2 vokalen und mindesten 2 motorischen Tics.

C mindestens 2 motorischen Tics.

D mindestens 2 motorischen Tics und mindestens einem vokalen Tic.

E mindestens 2 vokalen Tics.

\section{Frage 7}

Welche Intervention sollte zunächst nach Diagnosestellung erfolgen?

A Psychoedukation

B Antipsychotische Medikation

C Antidepressive Medikation

D Psychotherapie

E Ergotherapie

\section{Frage 8}

Welches Psychotherapieverfahren eignet sich in der Behandlung der primären Ticstörung/des Tourette-Syndroms?
A Analytische Psychotherapie
B Dialektisch-behaviorale Therapie
C Schematherpie
D Exposure and event prevention training
E Skillstraining 


\section{Punkte sammeln auf CME.thieme.de}

Fortsetzung ...

\section{Frage 9}

Welches Antipsychotikum ist am häufigsten verwendet in der Behandlung der primären Ticstörung/des Tourette-Syndroms?
A Haloperidol
B Olanzapin
C Flupentixol
D Aripiprazol
E Quetiapin

\section{Frage 10}

In welchen Fällen sollte eine tiefe Hirnstimulation als Therapieoption erwogen werden?
A Komorbide therapieresistente Depression
B Komorbider therapieresistente Zwangsgedanken
C Bei allen Patienten gleichermaßen empfehlenswert
D Nur bei therapiererefraktärem malignen Verlauf
E Nichtansprechen auf First-line-Antipsychotika 